\title{
Stam's Conjecture and Threshold Phenomena in Collision Resistance
}

\author{
John Steinberger ${ }^{1, \star}$, Xiaoming Sun ${ }^{2, \star \star}$, and Zhe Yang ${ }^{3}$ \\ 1 Institute of Theoretical Computer Science, Tsinghua University, Beijing \\ 2 Institute of Computing Technology, China Academy of Sciences \\ 3 Hulu Software, Beijing \\ \{jpsteinb, xiaoming.sun, yangzhe1990\}@gmail.com
}

\begin{abstract}
At CRYPTO 2008 Stam 8 conjectured that if an $(m+s)$ bit to $s$-bit compression function $F$ makes $r$ calls to a primitive $f$ of $n$-bit input, then a collision for $F$ can be obtained (with high probability) using $r 2^{(n r-m) /(r+1)}$ queries to $f$, which is sometimes less than the birthday bound. Steinberger 9] proved Stam's conjecture up to a constant multiplicative factor for most cases in which $r=1$ and for certain other cases that reduce to the case $r=1$. In this paper we prove the general case of Stam's conjecture (also up to a constant multiplicative factor). Our result is qualitatively different from Steinberger's, moreover, as we show the following novel threshold phenomenon: that exponentially many (more exactly, $2^{s-2(m-n) /(r+1)}$ ) collisions are obtained with high probability after $O(1) r 2^{(n r-m) /(r+1)}$ queries. This in particular shows that threshold phenomena observed in practical compression functions such as $\mathrm{JH}$ are, in fact, unavoidable for compression functions with those parameters.
\end{abstract}

\section{Introduction}

The ideal primitive model (IPM) is a popular paradigm in cryptographic security proofs. In this model one assumes that some primitive used by a construction, such as a blockcipher, is "ideal" - namely perfectly random subject to the constraints of the type of primitive under consideration - and one then bounds the chance of success of an adversary given oracle access to the ideal primitive, in some given security experiment, for some given number of queries. The adversary considered is almost always information-theoretic. As such, the adversary's only obstacle to achieving its attack is the randomness of the query responses.

Because the IPM considers information-theoretic adversaries certain limitations naturally arise as to what kind of security can be achieved for a certain

\footnotetext{
* Supported by the National Basic Research Program of China Grant 2011CBA00300, 2011CBA00301, the National Natural Science Foundation of China Grant 61033001, 61061130540, 61073174, and by NSF grant 0994380.

** Supported by the National Natural Science Foundation of China Grant 61170062, 61061130540, and the National Basic Research Program of China Grant 2011CBA00300, 2011CBA00301.
} 
functionality using a certain primitive a certain number of times. For example, consider the task of constructing a $2 n$-bit to $n$-bit compression function $F$ using a random $n$-bit to $n$-bit permutation $f$ as a primitive. There are $2^{2 n}$ inputs to $F$ but only $2^{n}$ inputs to $f$. Thus each input to $f$ corresponds on average to $2^{n}$ inputs to $F$, so with just two calls to $f$ we can learn to evaluate $F$ on at least $2 \cdot 2^{n}$ inputs. But this is more than the number of outputs of $F$, so a collision can be obtained with probability 1 in just two queries. Note that determining which two $f$-queries to make is no problem for an information-theoretic adversary, nor is "finding the collision" among the $2 \cdot 2^{n}$ mapped values. Thus it is not possible to design a compression function with these parameters that is collision resistant in the IPM.

This paper follows a line of work [2, 6, 8, 9, in the same vein as the above argument, seeking to establish the limits of provable security in the IPM model. Specifically, we focus the following question related to work of Stam [8] and, before that, of Rogaway and Steinberger [6] given $m, n, r, s \geq 1$, what is the maximum collision security of a compression function $F:\{0,1\}^{m+s} \rightarrow\{0,1\}^{s}$ that makes $r$ calls to an ideal primitive $f$ of domain $\{0,1\}^{n}$ ? (The range of $f$ is not specified because it turns out to be immateria 1.) Here "collision security" means the largest number of $f$-queries the best information-theoretic adversary can ask before achieving probability $\frac{1}{2}$ of obtaining a collision.

Since it costs at most $r$ queries to evaluate any point in the domain, a birthday attack implies that collision security cannot exceed $q=O(1) r 2^{s / 2}$ queries. However, depending on the parameters, other attacks may be more effective than birthday attacks. In particular Stam [8] conjectured that

$$
q=r\left\lceil 2^{(n r-m) /(r+1)}\right\rceil+1
$$

queries should always suffice for finding a collision with probability at least $\frac{1}{2}$. (We restate Stam's conjecture as slightly modified by Steinberger 9.) Roughly speaking, this bound is less than a birthday attack when $s / 2>(n r-m) /(r+$ $1)$. The latter occurs for example when $(m, n, r, s)=(n, n, 2, n)$, the case of a $2 n$-bit to $n$-bit compression function making two calls to a primitive of $n$-bit input, for which Stam's bound forecasts a maximum collision resistance of $2^{n / 3}$, which is more restrictive than the birthday bound of $2^{n / 2}$. As a second example, Stam's bound is even more restrictive when $(m, n, r, s)=(n, n, 1, n)$, for which it forecasts a maximum collision resistance of $O(1)$ queries; in fact this setting of parameters coincides with the first example discussed in the paper (regarding a compression function $F:\{0,1\}^{2 n} \rightarrow\{0,1\}^{n}$ making a single call to an $n$-bit random permutation).

Stam's conjecture is appealing because it apparently constitutes the optimal upper bound on collision resistance for all cases for which it beats the birthday bound, while the birthday bound can apparently be achieved in all other cases. In other words, as far as currently understood, it seems like the maximum collision

\footnotetext{
${ }^{1}$ Immaterial to proving the upper bound under consideration in this paper; better upper bounds on security should be provable if $f$ has sufficiently small range, see comments by Stam $[$.
} 
resistance of a compression function $F:\{0,1\}^{m+s} \rightarrow\{0,1\}^{s}$ making $r$ calls to a random function $f$ of $n$-bit input equals

$$
\min \left(r 2^{s / 2}, r\left\lceil 2^{(n r-m) /(r+1)}\right\rceil\right)
$$

up to possible lower order terms. This thesis is supported by a number of constructions [5, 7, 8].

Steinberger [9] obtained the only previous results on Stam's conjecture. He proved that when

$$
(2 m-n(r-1)) /(r+1) \geq 4.09
$$

$O(1) r\left\lceil 2^{(n r-m) /(r+1)}\right\rceil$ queries suffice to find a collision for $F$ with probability at least 0.5 . The condition (2) is increasingly restrictive as $r$ grows; for $r=1$, it reduces to $m \geq 4.09$; for $r=2$, it reduces to $\frac{2}{3} m-\frac{1}{3} n \geq 4.09$; for $r=3$ it reduces to $\frac{1}{2} m-\frac{1}{2} n \geq 4.09$; and so on. If $m=n$ (a typical case in real-world constructions) then (2) is false for all $r \geq 3$. Thus Stam's conjecture was until now, and despite Steinberger's result, very much open in the general case.

Steinberger also made the observation that for certain parameters $(m, n, r, s)$ Stam's conjecture can be reduced to parameters $\left(m^{\prime}, n, r^{\prime}, s\right)$ such that $m^{\prime}<m$ and $r^{\prime}<r$. (To be precise, such a reduction can be effected whenever $m r \geq$ $n$.) In fact, the core of Steinberger's result is a proof of Stam's conjecture for the case $2=1$ and $m \geq 4.09$. Other parameters $(m, n, r, s)$ with $r>1$ to which Steinberger's result applies are precisely those for which the reduced tuple $\left(m^{\prime}, n, r^{\prime}, s\right)$ has $r^{\prime}=1$ and $m^{\prime} \geq 4.09$ (inequality (2) is sufficient and necessary for both $r^{\prime}=1$ and $m^{\prime} \geq 4.09$ to hold). Thus Steinberger's result is "really" about the case $r=1$ of Stam's conjecture. (To be fair, the results of [9] nonetheless cover a large number of parameter settings of practical interest.)

In this paper we resolve the general case of Stam's conjecture. More precisely we show that if $F:\{0,1\}^{m+s} \rightarrow\{0,1\}^{s}$ is a compression function using $r$ calls to a primitive $f$ of $n$-bit input, where $m \geq 1$, then with high probability a collision can be found for $F$ in at most

$$
O(1) r\left\lceil 2^{\frac{n r-m}{r+1}}\right\rceil
$$

queries to $f$, where the $O(1)$ term represents a constant independent of all other parameters. This constant, being in the vicinity of 16000 , is large but not astronomical. Note that some lower bound must be imposed on $m$, since if $m=0$ the domain has the same size as the range, and $F$ may have no collisions at all (e.g., $F$ may ignore its primitive $f$, and be the identity on $\{0,1\}^{s}$ ). In fact, Stam's conjecture doesn't hold under the sole assumption $m>0$, as is easy to see. For example, this would allow the domain to have a single more point than the range, making a collision very hard to find 3 .

\footnotetext{
${ }^{2}$ When $m$ is not an integer, our meaning is that $F$ is a compression function of domain of size at least $\left\lceil 2^{s+m}\right\rceil$; the qualitative nature of the domain (be it bitstrings, or some other set) is not relevant. See Section 2 for a more precise statement.

${ }^{3}$ See Wiener [10] for details on the effectiveness of birthday attacks in functions were the size of the domain approaches the size of the range.
} 
At this point we emphasize that, like for Steinberger's theorem, the "primitive" $f$ called by the compression function $F$ can be any type of primitive of $n$-bit input, i.e., can be drawn from any distribution. Such a primitive can model, for example, a $n$-bit to $n$-bit permutation, but it can also model, say, a blockcipher 4 , or essentially any type of function-like primitive.

On the other hand our result is qualitatively different from Steinberger's in that we show an interesting threshold phenomenon: for the range of parameters in which Stam's bound is less than the cost of a birthday attack (namely, when $s / 2>(n r-m) /(r+1))$, we show many collisions are obtained with high probability as soon as $O(1) r\left\lceil 2^{\frac{n r-m}{r+1}}\right\rceil$ queries are made; more precisely, one obtains at least

$$
2^{s-\frac{2(n r-m)}{r+1}}
$$

collisions with high probability, using at most $16000 r\left\lceil 2^{\frac{n r-m}{r+1}}\right\rceil$ queries to $f$. In this regard, it is worth recalling that Stam's bound is conjecturally optimal (and for some settings of parameters provably optimal), implying that with only

$$
o(1) r\left\lceil 2^{\frac{n r-m}{r+1}}\right\rceil
$$

queries to $f$, no collisions for $F$ are found with high probability (presuming an adequate $F)$. Note the exponent $s-2(n r-m) /(r+1)$ in (3) is precisely twice the difference between the exponent in the cost of a birthday attack (a.k.a. $s / 2)$ and the exponent of Stam's conjecture (a.k.a. $(n r-m) /(r+1)$ ). Thus, the further Stam's bound is beneath the cost of a birthday attack, the sharper the threshold phenomenon.

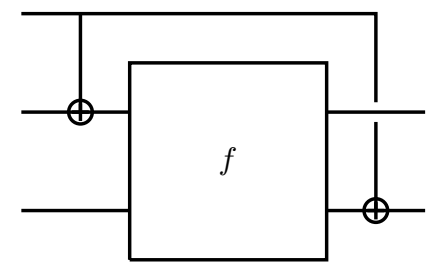

Fig. 1. The JH compression function $G:\{0,1\}^{1.5 n} \rightarrow\{0,1\}^{n}$. All wires carry $n / 2$-bit values.

As an example, we can consider the compression function $G$ of JH [1], one of the finalists in NIST's SHA-3 competition, pictured in Figure 1 This is a compression function from $\{0,1\}^{1.5 n}$ to $\{0,1\}^{n}$ using a single call to a primitive $f$ of $n$-bit input (more precisely, $f$ is a permutation). Thus the JH compression

\footnotetext{
${ }^{4}$ A blockcipher with $m$-bit word and $k$-bit key can be modeled as a primitive of input length $n=m+k$, or of input length $n=m+k+1$ if the construction also uses "inverse" blockcipher calls (in which case the extra bit indicates whether the call is forward or backward).
} 
function $G$ has parameters $(m, n, r, s)=(0.5 n, n, 1, n)$. The cost of a birthday attack for $G$ is $2^{n / 2}$. Stam's bound, however, is

$$
2^{\frac{n r-m}{r+1}}=2^{\frac{n-0.5 n}{2}}=2^{n / 4} .
$$

Thus Stam's conjecture indicates that the JH compression function $G$ must have significantly weaker than birthday collision resistance. It is indeed easy to see that, on average, only $2^{n / 4}$ queries are required to find a collision for $G$, since all one needs is to find a collision on the top half of output (the bottom half can then be adjusted via the input wire). Steinberger's theorem (and our own result as well) shows that any compression function with parameters $(m, n, r, s)=(0.5 n, n, 1, n)$, regardless of its design, will likewise have collision resistance at most $2^{n / 4}$ (up to a small constant factor). Observe, also, that once a single collision is obtained for $G, 2^{n / 2}$ collisions are obtained at once, since we can replicate the collision with any value on the bottom output wire. Our own theorem, beyond showing that collision resistance cannot exceed $2^{n / 4}$, predicts this threshold behavior as well. More precisely, we show that for any compression function with parameters $(m, n, r, s)=(0.5 n, n, 1, n)$, an adversary making at most5 $200 \cdot 2^{n / 4}$ queries to $f$ can obtain

$$
2^{s-\frac{2(n r-m)}{r+1}}=2^{n-\frac{2(n-0.5 n)}{2}}=2^{0.5 n}
$$

collision 6 with high probability. On the other hand, we emphasize that no collisions are obtained for $G$ with $\frac{1}{10} \cdot 2^{n / 4}$ queries (for any adversary, w.h.p.). Thus we not only pinpoint the collision resistance up to a constant factor, but we also pinpoint the exact "payoff" that occurs once collision resistance is breached.

As a second example, in 8] Stam exhibits a compression function with parameters $(m, n, r, s)=(n, n, 2, n)$ of collision resistance $2^{n / 3}$ (Stam's bound). Stam's compression function has the particularity that $n / 3$ bits are simply forwarded untouched from the input the output, whereas the remaining $2 n-n / 3=\frac{5}{3} n$ input bits are cryptographically processed into the remaining $n-n / 3=\frac{2}{3} n$ output bits. Obviously, for such a compression function, $2^{n / 3}$ collisions are obtained once a single collision is obtained. Our own result shows this sudden jump (from no collisions to $2^{n / 3}$ collisions) is essentially unavoidable, in the sense that with

$$
16000 \cdot 2^{\frac{n r-m}{r+1}}=16000 \cdot 2^{\frac{2 n-n}{3}}=16000 \cdot 2^{n / 3}
$$

queries an adversary can obtain

$$
2^{s-\frac{2(n r-m)}{r+1}}=2^{n-\frac{2(2 n-n)}{3}}=2^{n / 3}
$$

${ }^{5}$ When $r=1$ the multiplicative constant can be improved from 16000 to 200. See Section 3 for more details.

6 Traditionally, the "number of collisions" means the "number of distinct pairs of inputs that collide". Note, however, that under this definition $2^{0.5 n}$ "collisions" may be caused by only $2^{0.25 n}$ inputs, all involved in one big multi-collision. We show, in fact, that the number of different inputs involved in a collision is at least $2^{0.5 n}$, which constitutes an even stronger result. 
collisions with high probability, and this for any compression function with parameters $(m, n, r, s)=(n, n, 2, n)$.

Organization. Section 2 contains relevant definitions and conventions. Section 3 states and briefly discusses our main result. Section 4 gives an overview of the proof, and briefly compares our proof techniques to those of Steinberger [9]. The actual proof of our main theorem (this being Theorem 2 in Section 3 ) is left to the full version of this paper for reasons of space, but the key technical lemmas, which contain the more mathematically interesting techniques and on which the overview of Section 4 is also based, are proved in Appendix A.

\section{Definitions and Preliminaries}

Compression Functions. Let $m \geq 0$ be a real number and let $s \geq 0$ be an integer. (Our results hold as stated even when $s \geq 0$ is a real number such that $2^{s}$ is an integer and, likewise, also when $n \geq 0$ is a real number such that $2^{n}$ is an integer. However, for notational and conceptual simplicity, we shall assume $n, s$ are integers.) By "a function of domain $\{0,1\}^{s+m}$ " we mean a function with a domain of size $\left\lceil 2^{s+m}\right\rceil$ - the exact nature of the domain will not matter for our results, but for notational convenience we still write the domain as $\{0,1\}^{s+m}$ (even though $m$ is not necessarily an integer and, furthermore, even though $2^{s+m}$ is not necessarily an integer). Readers who feel uneasy about this convention may think of $\{0,1\}^{s+m}$ as being a shorthand for some fixed subset of $\{0,1\}^{\lceil s+m\rceil}$ of size $\left\lceil 2^{s+m}\right\rceil$.

Let now $m \geq 0$ be a real number and let $r \geq 1, n, s \geq 0$ be integers. We formalize the notion of a compression function $F:\{0,1\}^{s+m} \rightarrow\{0,1\}^{s}$ making $r$ calls to a primitive $f$ of domain $\{0,1\}^{n}$.

In fact we allow $F$ to call potentially distinct primitives $f_{1}, \ldots, f_{r}$ in fixed order mode, meaning $f_{i}$ is called before $f_{j}$ for $i<j$. Let $f_{1}, \ldots, f_{r}$ be (not necessarily distinct) functions of domain $\{0,1\}^{n}$ and range $\{0,1\}^{b}$, where $b$ is arbitrary. The compression function $F:\{0,1\}^{m+s} \rightarrow\{0,1\}^{s}$ is defined by $r$ functions $g_{1}, \ldots, g_{r}$ where $g_{i}:\{0,1\}^{m+s} \times\{0,1\}^{b(i-1)} \rightarrow\{0,1\}^{n}$ and a function $h:$ $\{0,1\}^{m+s} \times\{0,1\}^{b r} \rightarrow\{0,1\}^{s}$. We then define $F(v)=h\left(v, y_{1}, \ldots, y_{r}\right)$ where $y_{j}=$ $f_{j}\left(g_{j}\left(v, y_{1}, \ldots, y_{j-1}\right)\right)$ for $j=1 \ldots r$. We call the values $y_{1}, \ldots, y_{r}$ intermediate chaining variables and we refer to the functions $g_{1}, \ldots, g_{r}$ as the intermediate processing functions. We note that $g_{1}, \ldots, g_{r}$ are, for a given construction, fixed finite functions with a public description.

We say an adversary $A$ with oracle access to $f_{1}, \ldots, f_{r}$ "knows the first $k$ chaining variables" for some input $v \in\{0,1\}^{m+s}$ when $A$ has made the queries $f_{1}\left(g_{1}(v)\right)=y_{1}, f_{2}\left(g_{2}\left(v, y_{1}\right)\right)=y_{2}, \ldots, f_{k}\left(g_{k}\left(v, y_{1}, \ldots, y_{k-1}\right)\right)=y_{k}$, where $0 \leq$ $k \leq r$. In this case, we also say $A$ "knows the relevant queries to $f_{1}, \ldots, f_{k}$ " for $v$.

When $F$ is as defined above we call $F$ an " $(m, n, r, s)$ compression function". By default, the primitives called by such a compression function are always named $f_{1}, \ldots, f_{r}$ (in order).

Collision ACCOUnting. The following definition is somewhat nonstandard, but central to the paper: 
Definition 1. Let $F: D \rightarrow R$ be a function of domain $D$ and range $R$. Let $S \subseteq D$. The set of colliding inputs in $S$ (with respect to $F$ ) is the set

$$
\{x \in S: \exists y \in S, y \neq x, \text { s.t. } F(x)=F(y)\} .
$$

Let $F$ be an $(m, n, r, s)$ compression function calling primitives $f_{1}, \ldots, f_{r}$. Let $A$ be an adversary with oracle access to $f_{1}, \ldots, f_{r}$. The set of inputs learned by $A$ is the set of inputs $S \subseteq\{0,1\}^{s+m}$ for which $A$ has made the relevant queries to $f_{1}, \ldots, f_{r}$ at the end of its attack (and therefore, for which $A$ knows the value of $F)$. The set of colliding inputs obtained by $A$ is the set $C \subseteq S$ of colliding inputs in $S$, with respect to $F$. We say $A$ obtains $z$ colliding inputs if $|C| \geq z$.

It is worth noting that $|C| \geq|S|-|R|$, given that only $|R|$ elements of $S$ can occupy their "own" slots in the range. Thus an adversary that learns $|S|$ inputs for a compression function of range size $|R|$ automatically obtains at least $|S|-|R|$ colliding inputs.

YIELD. The following basic observation is due to Rogaway and Steinberger [6]:

Lemma 1. Let $F:\{0,1\}^{m+s} \rightarrow\{0,1\}^{s}$ be a compression function calling primitives $f_{1}, \ldots, f_{r}:\{0,1\}^{n} \rightarrow\{0,1\}^{b}$ in fixed-order mode. Then there exists an adversary that with at most $q$ queries to each $f_{i}$ can learn the first $i$ intermediate chaining variables for at least

$$
2^{m+s}\left(\frac{q}{2^{n}}\right)^{i}
$$

inputs, for $0 \leq i \leq r$.

In other words, there exists an adversary making at most $q$ queries to each $f_{i}$, and for which

$$
\left|S_{i}\right| \geq 2^{m+s}\left(\frac{q}{2^{n}}\right)^{i}
$$

for $0 \leq i \leq r$, where $S_{i} \subseteq\{0,1\}^{m+s}$ is the set of inputs for which the relevant queries to $f_{1}, \ldots, f_{i}$ have been made. The adversary in question is very straightforward: it is a greedy adversary that starts by choosing its queries to $f_{1}$ such as to maximize the size of $S_{1}$, then, after making its queries to $f_{1}$, chooses its queries to $f_{2}$ such as to maximize the size of $S_{2} \subseteq S_{1}$, and so on. For a full proof see any of [6], [8] or [9].

Setting $i=r$ in Lemma 1 we obtain the following corollary:

Corollary 1. Let $F:\{0,1\}^{m+s} \rightarrow\{0,1\}^{s}$ be a compression function calling primitives $f_{1}, \ldots, f_{r}:\{0,1\}^{n} \rightarrow\{0,1\}^{b}$ in fixed-order mode. Then with q queries to each $f_{i}$, an adversary can learn to evaluate $F$ on at least

$$
2^{m+s}\left(\frac{q}{2^{n}}\right)^{r}
$$

inputs. 


\section{Results}

The following Theorem dispatches the "easy" cases of Stam's conjecture; similar results are already given in 6, 8, 9 .

Theorem 1. (cf. [6, 8, 9]) Let $F$ be an $(m, n, r, s)$ compression function with $m \geq 1$. Then: (i) if $s / 2 \leq(n r-m) /(r+1)$, a collision can be found for $F$ with at most

$$
q=2 \sqrt{2} \cdot 2^{s / 2}+1 \leq 2 \sqrt{2} \cdot 2^{\frac{n r-m}{r+1}}+1
$$

queries to each $f_{i}$, with probability at least 0.5 ; and (ii) if $m \geq n r$, a collision can be found for $F$ with at most 2 queries to each $f_{i}$, with probability 1.

Proof. Statement (i) follows by a birthday attack and the fact that $m \geq 1$ (so that the domain of $F$ has size at least twice the range); see [9,10] for more details. Statement (ii) follows from by applying Corollary 1 with $q=2$, and noting that when $m \geq n r$ we have

$$
2^{m+s}\left(\frac{2}{2^{n}}\right)^{r} \geq 2 \cdot 2^{s}
$$

so that, automatically, at least $2^{s+1}-2^{s}=2^{s}$ colliding inputs are obtained by the adversary.

In light of Theorem 1, our remaining results are restricted to the case $(s / 2 \geq$ $(n r-m) /(r+1) \wedge m \leq n r)$. It is worth noting that $2^{(n r-m) /(r+1)} \geq 1$ when $m \leq n r$, since then $(n r-m) /(r+1) \geq 0$.

To state and discuss our main result it will be convenient to define the function

$$
\gamma(r, c)=2 e^{-c^{2} / 5760}+\sum_{i=1}^{r-1} 2 e^{-\frac{1}{32}\left(\frac{c}{80}\right)^{i}}
$$

where $r \geq 1$ is an integer and $c>0$ is an arbitrary real number. We keep this definition of $\gamma(r, c)$ for the rest of the paper.

Our main result is the following:

Theorem 2. Let $F$ be an $(m, n, r, s)$ compression function with $1 \leq m \leq n r$ and $s / 2 \geq(n r-m) /(r+1)$. Let $c>0$ be a real number such that $c 2^{\frac{n r-m}{r+1}}$ is an integer. Then there exists an adversary making at most

$$
q=2 c 2^{\frac{n r-m}{r+1}}
$$

queries to each $f_{i}$ and obtaining at least

$$
2^{s-\frac{2(n r-m)}{r+1}}
$$

colliding inputs, with probability at least $1-\gamma(r, c)$. 
For $r=1$ one can compute that $\gamma(1,90)<0.5$, whereas $\gamma(1,100)<0.36$ and $\gamma(1,1000)<e^{-170}$. Thus $180\left\lceil 2^{\frac{n r-m}{r+1}}\right\rceil$ queries suffice to obtain a collision with probability at least 0.5 . For $r>1$, one can make the observation that

$$
\gamma(r, c) \leq 2 e^{-c^{2} / 5760}+\sum_{i=1}^{\infty} 2 e^{-\frac{1}{32}\left(\frac{c}{80}\right)^{i}}
$$

where the right-hand side does not depend on $r$, and where the right-hand side is less than 0.5 for $c \geq 8000$. Thus $16000 r\left\lceil 2^{\frac{n r-m}{r+1}}\right\rceil$ queries (in total to all $f_{i}$ 's) suffice to find a collision with probability at least 0.5 when $r>1$.

The proof of Theorem 2 is left to the paper's full version. However the main ideas behind the proof are presented in the next Section, with supporting lemmas in Appendix $\mathrm{A}$

\section{Proof Overview}

In this section we give an overview of the proof of Theorem 2 . We emphasize that this section's contents constitute intuition only and have little mathematical value. An independent, fully self-contained proof of Theorem 2 appears in the paper's full version. Nonetheless, the more technical lemmas needed to implement the ideas described below are proved in this version, in Appendix A

A central ingredient in our proof is a lemma on collisions (Lemma 5 in Appendix A that we start by paraphrasing here in order to facilitate the following discussion. Let $T_{1}, \ldots, T_{k}$ be disjoint sets whose sizes are upper bounded by some constant $M$, let $F: T \rightarrow R$ be some function where $T=T_{1} \cup \cdots \cup T_{k}$, and let $C$ be the total number of colliding inputs in $T$ with respect to $F$. Note that if we select $q$ of the $k$ sets $T_{1}, \ldots, T_{k}$ at random, and form a set $T^{\prime}$ as the union of the $q$ selected sets, then each point of $T$ has probability $p:=q / k$ of ending up in $T^{\prime}$. Since a colliding input $x_{0} \in T$ has probability at least

$$
\frac{q}{k} \frac{q-1}{k-1} \approx p^{2}
$$

of winding up as a colliding input in $T^{\prime}$ (because $x_{0}$ must be selected for $T^{\prime}$ and also at least one of the other point: 7 in $T$ with which $x_{0}$ collides must be selected for $T^{\prime}$ ), we can therefore expect $T^{\prime}$ to have approximately at least

$$
p^{2} C
$$

colliding inputs. Roughly speaking, Lemma 5 states that as long as this expectation is a fair amount larger than $M$ (the maximum size of the $T_{i}$ 's) then this intuition is borne out, and the number of colliding inputs in $T^{\prime}$ is not much less that $p^{2} C$ with high probability. We point out that Lemma [5 does not "know"

\footnotetext{
${ }^{7}$ If such an other point comes from the same set $T_{i}$ that contains $x_{0}$, this only helps us, in the sense that $x_{0}$ then has chance exactly $p$ (the chance that $T_{i}$ is selected) of becoming a colliding input in $T^{\prime}$.
} 
how to take advantage of multi-collisions: if a colliding input $x_{0} \in T$ collides with very many other points in $T$, coming from many different $T_{j}$ 's, then $x_{0}$ 's chance of being a colliding input in $T^{\prime}$ will be significantly greater than $p^{2}$. Thus Lemma 5 does not give a sharp result in all situations. This lack plays a role in the proof sketch below (as well as in the proof itself).

For the proof sketch we start by reviewing some specific settings of the parameters and explain, in each case, how our collision-finding adversary operates, and why it can hope to find the desired number of collisions within the limits of Stam's bound. We call these "case studies". We later abstract more general observations from these case studies. (The first two case studies concern parameter settings that are already covered by Steinberger's 9] results. However, the point is to flesh out our line of attack, which is completely different from Steinberger's birthday-based approach.)

Conceptually we emphasize that, unlike for a typical collision resistance analysis in the provable security setting, it is more useful to view the primitives $f_{1}, \ldots, f_{r}$ as being sampled (from whatever distribution) before the start of the collision resistance experiment, rather than as being lazy sampled. Thus one can think of the primitives $f_{1}, \ldots, f_{r}$ as functions that are "fixed but arbitrary", and to which the adversary has oracle access.

First Case Study: $(m, n, r, s)=(0.5 n, n, 1, n)$. Let $F:\{0,1\}^{m+s} \rightarrow\{0,1\}^{n}$ be a compression function making a single call to an $n$-bit primitive $f_{1}$, where $(m, n, r, s)=(0.5 n, n, 1, n)$. Thus $F:\{0,1\}^{1.5 n} \rightarrow\{0,1\}^{n}$. We note this setting of parameters coincides, for example, with the parameters of the JH compression function (discussed in the introduction).

Stam's bound indicates that

$$
q=2^{\frac{n r-m}{r+1}}=2^{\frac{n-0.5 n}{2}}=2^{n / 4}
$$

queries to $f_{1}$ should suffice to find collisions for $F$. Let $S_{0}=\{0,1\}^{m+s}$ be $F^{\prime}$ 's domain, and write

$$
S_{0}=\bigcup_{y \in\{0,1\}^{n}} U_{y}^{0}
$$

where

$$
U_{y}^{0}=\left\{x \in S_{0}: g_{1}(x)=y\right\}
$$

where $g_{1}$ is $F$ 's first and only intermediate processing function (see Section 2). We note that $S_{0}$ is the disjoint union of the sets $U_{y}^{0}$. Moreover, the collision adversary knows each set $U_{y}^{0}$, since $g_{1}$ is public. We also note that the average size of the sets $\left\{U_{y}^{0}: y \in\{0,1\}^{n}\right\}$ is

$$
\frac{\left|S_{0}\right|}{2^{n}}=\frac{2^{m+s}}{2^{n}}=\frac{2^{1.5 n}}{2^{n}}=2^{n / 2} .
$$

For simplicity we start by assuming that $\left|U_{y}^{0}\right|=2^{n / 2}$ for all $y \in\{0,1\}^{n}$. We will discuss later how to lift this assumption. 
The adversary's most natural strategy is to make $2^{n / 4}$ random queries to $f_{1}$. Let $\mathcal{B} \subseteq 2^{n / 4}$ denote the set of values so queried to $f_{1}$, and set

$$
S_{1}=\bigcup_{y \in \mathcal{B}} U_{y}^{0} .
$$

Then $S_{1} \subseteq\{0,1\}^{m+s}$ is the set of inputs for which the relevant query to $f_{1}$ is known. Note that $\left|S_{1}\right|=2^{n / 4} \cdot 2^{n / 2}=2^{3 n / 4}$ by our assumption that each set $U_{y}^{0}$ has size $2^{n / 2}$.

We could try, at this point, to estimate the number of colliding inputs in $S_{1}$ using Lemma [5] applied with $T=S_{0}$ and $T^{\prime}=S_{1}$, where the sets $T_{1}, \ldots, T_{k}$ correspond to the family of sets $\left\{U_{y}^{0}: y \in\{0,1\}^{n}\right\}$ (which form a disjoint partition of $S_{0}$ ), and where $M=2^{n / 2}$ is the upper bound on the size of the $T_{i}$ 's. Here $k=2^{n}$ and, therefore, $p=q / k=2^{n / 3} / 2^{n}=2^{-2 n / 3}$. The number of colliding inputs $C$ in $T=S_{0}$ is at least $\left|S_{0}\right|-2^{s}=2^{1.5 n}-2^{n} \approx 2^{1.5 n}$. We therefore find that

$$
p^{2} C \approx 2^{-4 n / 3} 2^{1.5 n}=2^{n / 6} .
$$

Unfortunately, this number is not as large as $M=2^{n / 2}$ and, in such a case, Lemma 5 does not deliver anything meaningful. We are running up against the afore-mentioned shortcoming of Lemma 5] since we are in a case where the average colliding input in $T=S_{0}$ does not only collide with $O(1)$ other elements in $T$, but with very many other elements (or more precisely with $\left|S_{0}\right| / 2^{s}=2^{n / 2}$ other elements).

We overcome this obstacle with a trick. We divide the adversary's querying process into two phases. In the first phase, the adversary selects (deterministically, say) a subset $I$ of $\{0,1\}^{n}$ of size $2^{n / 2+1}$. In the second phase, the adversary selects a set $\mathcal{B} \subseteq I$ of size $2^{n / 4}$ uniformly from all such subsets of $I$, and queries the elements of $\mathcal{B}$ to $f_{1}$. We emphasize that the elements of $I$ not in $\mathcal{B}$ are not queried to $f_{1}$. Clearly, applying this two-step process is equivalent to directly selecting $2^{n / 4}$ values $\mathcal{B}$ uniformly at random from $\{0,1\}^{n}$ and querying them to $f_{1}$.

Let

$$
S_{I}=\bigcup_{y \in I} U_{y}^{0} .
$$

Thus $S_{0} \supseteq S_{I} \supseteq S_{1}$. Moreover $\left|S_{I}\right|=2^{n / 2+1} \cdot 2^{n / 2}=2^{n+1}=2^{s+1}$, so $S_{I}$ contains at least $2^{s+1}-2^{s}=2^{s}$ colliding inputs. (Note, crucially, that every colliding input in $S_{I}$ might very well collide with only one other input in $S_{I}$, so that we are no longer in a case in which Lemma 5 is ignoring a key statistic.) We now apply Lemma 5 with $T=S_{I}$ and $T^{\prime}=S_{1}$, where the sets $T_{1}, \ldots, T_{k}$ correspond, this time, to the family of sets $\left\{U_{y}^{0}: y \in I\right\}$ (which form a partition of $S_{I}$ ). Thus $k=|I|=2^{n / 2+1}$. We have $q / k=2^{n / 4} / 2^{n / 2+1} \approx 2^{-n / 4}$ and

$$
p^{2} C \approx 2^{-2 n / 4} 2^{s}=2^{n / 2}
$$

where $C \geq 2^{s}$ is the number of colliding inputs in $S_{I}$. Thus, this time, $p^{2} C$ is commensurate with the upper bound $M=2^{n / 2}$ on the size of the $T_{i}$ 's, and so 
Lemma 5 can be effectively applied. (To be a little more precise, by making, say, $200 \cdot 2^{n / 2}$ queries to $f_{1}$ instead of $2^{n / 2}$ queries to $f_{1}$, we can push $C$ and $p^{2} C$ to significantly higher than $M$, which remains capped at $2^{n / 2}$. Moreover we can make $\frac{1}{20} p^{2} C \geq 2^{n / 2}$ so that, by Lemma 5. we actually obtain $2^{n / 2}$ colliding inputs with high probability.)

We emphasize that the above argument does not require $f_{1}$ to be "random" at all; $f_{1}$ can be any fixed function. The only randomness occurs in the selection of the set $\mathcal{B}$ of queries to $f_{1}$.

Finally, the "well-balancedness" assumption on the sets $U_{y}^{0}$ can be removed by using a common refinement of these sets. More precisely, by Lemma 6] in Appendix $\underline{\mathrm{A}}$, one can always refine the collection of sets $\left\{U_{y}^{0}: y \in\{0,1\}^{n}\right\}$ into a collection of sets each of size at most $2^{n / 2}$, at the cost of increasing the number of sets by a factor of at most 2 . We then view the adversary as "querying" sets in this refinement (each such set is a subset of a particular $U_{y}^{0}$ and, therefore, associated to a particular value of $y$ ). This process may result in redundant queries to $f_{1}$ (when two or more subsets of the same $U_{y}^{0}$ are chosen to be queried), but this is harmless. In particular, we do not care about the fact that such redundant queries to $f_{1}$ produce dependent results - indeed, from the proof's standpoint, $f_{1}$ is anyway an arbitrary fixed function containing no entropy.

Second Case Study: $(m, n, r, s)=(n, n, 2, n)$. Let $F:\{0,1\}^{m+s} \rightarrow\{0,1\}^{n}$ be a compression function making calls to two $n$-bit primitives $f_{1}$ and $f_{2}$ in fixedorder mode, where $(m, n, r, s)=(n, n, 2, n)$. Thus $F:\{0,1\}^{2 n} \rightarrow\{0,1\}^{n}$. As usual, let $g_{1}$ and $g_{2}$ be the intermediate processing functions for $F$.

Stam's bound forecasts a collision resistance of

$$
q=2^{\frac{n r-m}{r+1}}=2^{\frac{2 n-n}{3}}=2^{n / 3}
$$

queries to each $f_{1}$ and $f_{2}$.

Let $S_{0}=\{0,1\}^{m+s}$ and let

$$
U_{y}^{0}=\left\{x \in S_{0}: g_{1}(x)=y\right\} .
$$

The adversary starts by querying $f_{1}(y)$ for the $q$ values $y$ for which $\left|U_{y}^{0}\right|$ is largest. (Note this is a deterministic step.) Then

$$
\left|S_{1}\right| \geq 2^{m+s}\left(\frac{q}{2^{n}}\right)=2^{2 n} \frac{2^{n / 3}}{2^{n}}=2^{4 n / 3}
$$

where $S_{1} \subseteq\{0,1\}^{m+s}$ is the set of inputs for which the relevant query to $f_{1}$ has been made. Note that $2^{4 n / 3} \gg 2^{n}=2^{s}$, so $S_{1}$ contains many colliding inputs (more precisely, at least $2^{4 n / 3}-2^{n} \approx 2^{4 n / 3}$ ) with probability 1 . Moreover, depending on the structure of $F$ and of $f_{1}$, there is no reason one could expect to beat this number of colliding inputs (in $S_{1}$ ) by using a randomized query strategy to $f_{1}$ instead of a greedy query strategy to $f_{1}$.

For simplicity, we will assume that $S_{1}$ has size exactly $2^{4 n / 3}$ (anyway the adversary could choose to "throw out" or ignore elements of $S_{1}$ to reduce the effective size of $S_{1}$ to $2^{4 n / 3}$, if desired). 
At this point, before queries to $f_{2}$ are made, note that we are essentially reduced to attacking a compression function $F^{\prime}$ with paramaters $\left(m^{\prime}, n^{\prime}, r^{\prime}, s^{\prime}\right)=$ $(n / 3, n, 1, n)$ whose domain is $S_{1}$, where $\left|S_{1}\right|=2^{m^{\prime}+s^{\prime}}=2^{4 n / 3}$. For such a compression function, Stam's bound quotes a collision resistance of

$$
2^{\frac{n^{\prime} r^{\prime}-m^{\prime}}{r^{\prime}+1}}=2^{\frac{n-n / 3}{2}}=2^{n / 3}
$$

queries, which is exactly our budget query for $f_{2}$. We have thus reduced the parameter setting $(m, n, r, s)=(n, n, 2, n)$ of Stam's conjecture to the parameter setting $(n / 3, n, 1, n)$, namely to a case of Stam's conjecture where $r=1$. (This type of reduction was first brought to attention by Steinberger [9.) What follows is therefore fairly similar to the first case study for the parameters $(m, n, r, s)=$ $(0.5 n, n, 1, n)$.

Let

$$
U_{y}^{1}=\left\{x \in S_{1}: g_{2}\left(x, y_{1}\right)=y\right\}
$$

for each $y \in\{0,1\}^{n}$, where, above, $y_{1}=f_{1}\left(g_{1}(x)\right)$ is the first intermediate chaining variable for $x$ (implicitly dependent on $x$ ). For simplicity, we can assume that $\left|U_{y}^{1}\right|=\left|S_{1}\right| / 2^{n}=2^{n / 3}$ for all $y \in\{0,1\}^{n}$ (this assumption can be lifted by using a refinement of the $U_{y}^{1}$ 's, as in the previous case study). To make his queries to $f_{2}$, the adversary starts by (deterministically) selecting a set $I \subseteq\{0,1\}^{n}$ of size $2^{2 n / 3+1}$. Let

$$
S_{I}=\bigcup_{y \in I} U_{y}^{1}
$$

so that $\left|S_{I}\right|=2^{2 n / 3+1} \cdot 2^{n / 3}=2^{n+1}=2^{s+1}$. Thus $S_{I}$ contains at least $2^{s}$ colliding inputs. The adversary then selects a random subset $\mathcal{B}$ of $I$ of size $q=2^{n / 3}$, and queries $f_{2}$ at all the points in $\mathcal{B}$. Let

$$
S_{2}=\bigcup_{y \in \mathcal{B}} U_{y}^{1}
$$

so that $S_{2} \subseteq S_{1}$ is the set of inputs for which the relevant queries to $f_{1}$ and $f_{2}$ are both known. Applying Lemma 5 with $T=S_{1}, T^{\prime}=S_{2}$, and with sets $T_{1}, \ldots, T_{k}$ corresponding to $\left\{U_{y}^{1}: y \in I\right\}$, where $k=|I|=2^{2 n / 3+1}$ and $\left|T_{i}\right| \leq M:=2^{n / 3}$ for all $i$, we find that $p=q / k=2^{n / 3} / 2^{2 n / 3+1} \approx 2^{-n / 3}$ and

$$
p^{2} C \approx 2^{-2 n / 3} 2^{s}=2^{n / 3}
$$

where $C \geq 2^{s}$ is the number of colliding inputs in $S_{I}$. Since the latter quantity is commensurate with $M$, we can effectively apply Lemma 5 to conclude that we will obtain $2^{n / 3}$ colliding inputs in $S_{2}$ with high probability (by making some constant factor more queries than $2^{n / 3}$ ).

Third Case Study: $(m, n, r, s)=(1.25 n, n, 4,2 n)$. Let $F:\{0,1\}^{m+s} \rightarrow\{0,1\}^{n}$ be a compression function making calls to four $n$-bit primitives $f_{1}, \ldots, f_{4}$ in fixed-order mode, where $(m, n, r, s)=(1.25 n, n, 4,2 n)$. In this case, therefore, $F:\{0,1\}^{3.25 n} \rightarrow\{0,1\}^{2 n}$. 
Stam's bound places collision resistance at

$$
q=2^{\frac{n r-m}{r+1}}=2^{\frac{4 n-1.25 n}{5}}=2^{0.55 n}
$$

queries to each of the primitives $f_{1}, f_{2}, f_{3}$ and $f_{4}$ (which, we note, is less than the cost of a birthday attack).

Let $S_{0}=\{0,1\}^{m+s}$ and let $U_{y}^{0}=\left\{x \in S_{0}: g_{1}(x)=y\right\}$ for all $y \in\{0,1\}^{n}$. The adversary starts by querying $f_{1}(y)$ for the $q$ values $y$ for which $\left|U_{y}^{0}\right|$ is largest. Then

$$
\left|S_{1}\right| \geq 2^{m+s}\left(\frac{q}{2^{n}}\right)=2^{3.25 n} \frac{2^{0.55 n}}{2^{n}}=2^{2.8 n}
$$

where $S_{1}$ is the set of inputs for which queries to $f_{1}$ have been made.

After the queries to $f_{1}$ are completed, let $U_{y}^{1}=\left\{x \in S_{1}: g_{2}\left(x, y_{1}\right)=y\right\}$ where $y_{1}$ is the first intermediate chaining variable for $x$. For $f_{2}$ the adversary again makes greedy queries, i.e. queries $f_{2}(y)$ for the $q$ values $y$ for which $\left|U_{y}^{1}\right|$ is largest. Then

$$
\left|S_{2}\right| \geq\left|S_{1}\right|\left(\frac{q}{2^{n}}\right)=2^{2.8 n} \frac{2^{0.55 n}}{2^{n}}=2^{2.35 n}
$$

where $S_{2} \subseteq S_{1}$ is the set of inputs for which queries to both $f_{1}$ and $f_{2}$ have been made. Note $S_{2}$, like $S_{1}$ is still larger than $2^{s}=2^{2 n}$; thus we are "automatically" assured the presence of colliding inputs in $S_{1}$ and $S_{2}$ by virtue of the size of these sets, which accounts for the sufficiency of the greedy approach.

After the queries to $f_{2}$ are completed, let $U_{y}^{2}=\left\{x \in S_{2}: g_{3}\left(x, y_{1}, y_{2}\right)=y\right\}$, where $y_{1}, y_{2}$ are the first two intermediate chaining variables for $x$. At this point, if the adversary were again to apply a greedy strategy for $f_{3}$, we would find a lower bound of

$$
\left|S_{2}\right|\left(\frac{q}{2^{n}}\right)=2^{2.35 n} \frac{2^{0.55 n}}{2^{n}}=2^{1.9 n}
$$

on the size of $S_{3}$, which is no longer larger than $2^{s}$. Applying a (deterministic) greedy strategy would therefore be a very bad idea, since one could easily set up $F$ and its primitives $f_{1}, \ldots, f_{4}$ so that $S_{3}$ contains no colliding inputs with probability 1 , and the adversary finds collisions with probability 0 .

Instead, at this stage we revert to using Lemma 5 and the two-step "trick" involving the set $I$. Assume for simplicity (and in fact without loss of generality) that $\left|U_{y}^{2}\right|=2^{2.35 n} / 2^{n}=2^{1.35 n}$ for all $y \in\{0,1\}^{n}$. The adversary starts by deterministically selecting a set $I \subseteq\{0,1\}^{n}$ of size $2^{0.65 n+1}$. Let

$$
S_{I}=\bigcup_{y \in I} U_{y}^{2}
$$

Thus $\left|S_{I}\right|=2^{0.65 n+1} 2^{1.35 n}=2^{2 n+1}=2^{s+1}$. (We note the adversary has been deterministic up to now - namely the adversary remains deterministic as long as the underlying set of known inputs contains colliding inputs simply by virtue of its size. Now the adversary is about to switch to, and stick with, a randomized 
strategy.) The adversary then randomly selects a set $\mathcal{B} \subseteq I$ of size $q=2^{0.55 n}$, and queries these values to $f_{3}$. We set

$$
S_{3}=\bigcup_{y \in \mathcal{B}} U_{y}^{2}
$$

We apply Lemma [5 with $T=S_{I}, T^{\prime}=S_{3},\left\{T_{i}: 1 \leq i \leq k\right\}=\left\{U_{y}^{2}: y \in I\right\}$, $k=|I|=2^{0.65 n+1}, M=2^{1.35 n}, p=q / k=2^{0.55 n} / 2^{0.65 n+1} \approx 2^{-0.1 n}$ and $C \geq\left|S_{I}\right|-2^{s} \geq 2^{s}=2^{2 n}$, so that

$$
p^{2} C \geq 2^{-0.2 n} 2^{2 n}=2^{1.8 n} .
$$

In particular, $p^{2} C \gg M$, so Lemma [5 can be effectively applied to show that the number of colliding inputs in $S_{3}$ is not much less than $p^{2} C=2^{1.8 n}$. For simplicity, we will assume the number of colliding inputs in $S_{3}$ is exactly $2^{1.8 n}$. Moreover, note that $\left|S_{3}\right|=2^{1.35 n} 2^{0.55 n}=2^{1.9 n}$ by virtue of our assumption that $\left|U_{y}^{2}\right|=2^{1.35 n}$ for each $y \in\{0,1\}^{n}$.

For queries to $f_{4}$, the adversary directly continues with a randomized strategy and an application of Lemma 5-no need for a preliminary selection of inputs $I$, here, because the number of colliding inputs in $S_{3}$ is already less than $2^{s}$.

More precisely, let $U_{y}^{3}=\left\{x \in S_{3}: g_{4}\left(x, y_{1}, y_{2}, y_{3}\right)=y\right\}$ for all $y \in\{0,1\}^{n}$, where $y_{1}, y_{2}, y_{3}$ are the intermediate chaining variables for $x$. Assume for simplicity that $\left|U_{y}^{3}\right|=\left|S_{3}\right| / 2^{n}=2^{1.9 n} / 2^{n}=2^{0.9 n}$ for all $y$. The adversary selects a random set $\mathcal{B} \subseteq\{0,1\}^{n}$ of size $q=2^{0.55 n}$, and queries these values to $f_{4}$. Set

$$
S_{4}=\bigcup_{y \in \mathcal{B}} U_{y}^{3} .
$$

We apply Lemma 5 with $T=S_{3}, T^{\prime}=S_{4},\left\{T_{i}: 1 \leq i \leq k\right\}=\left\{U_{y}^{3}: y \in\{0,1\}^{n}\right\}$, $k=|I|=2^{n}, M=2^{0.9 n}, p=q / k=2^{0.55 n} / 2^{n}=2^{-0.45 n}$ and $C=2^{1.8 n}$, where the latter equality comes from our simplifying assumption that $S_{3}$ contains exactly $2^{1.8 n}$ colliding inputs. Then

$$
p^{2} C=2^{-0.9 n} 2^{1.8 n}=2^{0.9 n}
$$

so that $p^{2} C$ is commensurate with $M=2^{0.9 n}$, and Lemma 5 can be effectively applied (after, potentially, multiplying the number of queries by some small constant) to show that at least

$$
p^{2} C=2^{0.9 n}=2^{2 n-1.1 n}=2^{s-\frac{2(n r-m)}{r+1}}
$$

colliding inputs can be obtained with good probability.

Digest. The last case study exhibits more or less all the features of the general case. In the general case, the adversary's querying strategy has two phases. The first phase is a "deterministic" phase where the adversary makes greedy queries to maximize the yield. This phase lasts as long the next set $S_{i}$ obtained is guaranteed to be larger than $2^{s}$. This phase also "spills over" into the (still deterministic) selection of the set $I$. The second phase then commences, consisting 
of purely random queries. (First $q$ random queries selected from $I$ and then, for subsequent $f_{i}$ 's, $q$ random queries selected from $\{0,1\}^{n}$.) It so turns out that the "phase change" occurs exactly when it is time to make queries to $f_{r_{0}+1}$ where

$$
r_{0}=\left\lfloor\frac{m(r+1)}{m+n}\right\rfloor .
$$

Thus, in the general case, the two-phase strategy determines a sequence of sets

$$
S_{0} \supseteq S_{1} \supseteq \cdots \supseteq S_{r_{0}} \supseteq S_{I} \supseteq S_{r_{0}+1} \supseteq \cdots \supseteq S_{r}
$$

where $S_{0}=\{0,1\}^{m+s}$ is $F$ 's domain and $S_{i}, i \geq 1$, is the set of inputs for which the queries to $f_{1}, \ldots, f_{i}$ have been made. (When $\frac{m(r+1)}{m+n}$ happens to be an integer - which does not occur in any of the case studies above - then $r_{0}=$ $\frac{m(r+1)}{m+n} \geq 1$ and, by adding a constant factor to the number of queries, one finds $\left|S_{r_{0}}\right| \geq 2^{s+1}$ instead of $\left|S_{r_{0}}\right|=2^{s}$, so that there is "still room" for $S_{I}$ to be selected.)

One can point out that the number of colliding inputs in the sets $S_{0}, \ldots, S_{r}$ evolves differently during the first and second phases. During the first phase, each colliding input in $S_{i}$ collides on average with a very large number of other points, so that the key factor determining whether a colliding input makes it from $S_{i}$ to $S_{i+1}$ (assuming $i+1 \leq r_{0}$ ) is just whether that particular point makes it to $S_{i+1}$ (since it is very likely that at least one of the myriad other points it collides with has made it to $S_{i+1}$ as well). The "rate of attrition" of colliding inputs is therefore $p=q / 2^{n}$ in going from $S_{i}$ to $S_{i+1}$, for $i+1 \leq r_{0}$, and, similarly, is $|I| / 2^{n}$ in going from $S_{r_{0}}$ to $S_{I}$. During the second phase, on the other hand, both a colliding input and the (on average unique) other input it collides with must simultaneously survive the selection process, so that the rate of attrition of colliding inputs in going from $S_{I}$ to $S_{r_{0}+1}$ is $(q /|I|)^{2}$ whereas the rate of attrition in going from $S_{i}$ to $S_{i+1}$ is $\left(q / 2^{n}\right)^{2}$ for $r_{0}+1 \leq i \leq r-1$. It is possible to compute that these rates of attrition lead to a final expected number of colliding inputs equal to $2^{s}-\frac{2(n r-m)}{r+1}$. The latter also equals, by no coincidence, $\left|S_{r-1}\right| / 2^{n}$.

Comparison with [9]. The proof of Lemma [5 our paper's "key lemma"uses ideas from Steinberger's "MECMAC lemma" 9] (a lemma which is actually unused in the main result of [9]), and more precisely recycles the nice idea of using a bipartition of sets to overcome dependencies between collision events. Our work also uses Steinberger's parameter reduction idea (as discussed in the second case study). However, these are essentially the only similarities with 9 . In particular, our proof does not consist in a generalization of Steinberger's techniques, since our proof, as restricted to $r=1$, does not reduce to a birthday attack, but instead uses Lemma 5 which itself relies on Martingale concentration results. Moreover, the key idea of focusing on the number of colliding inputs (as opposed to the more usual "number of colliding pairs of inputs") as the correct metric for measuring the progress of an attack is an original contribution of this paper. 


\section{Future Work}

Many related interesting open problems remain. One of the basic questions that remains is to show Stam's bound is tight. This would require exhibiting an infinite class of compression functions (parameterized by $m, n, r, s$, where $m$, $r$ and $s$ are linear functions of $n$ ) whose collision resistance is provably in the vicinity of

$$
\min \left(2^{s / 2},\left\lceil 2^{\frac{n r-m}{r+1}}\right\rceil\right) .
$$

Another remaining open question concerns parallelism. Could better attacks be found for compression functions that call their primitives in parallel? So far, rather amazingly, we are not aware of any provable separation between the power of parallel and sequential compression functions. A third type of question concerns adapting results like those in this paper to compression functions with primitives of not-all-equal input lengths and, maybe more interestingly, to primitives with small output lengths. Indeed, primitives with small output lengths constitute a vulnerability, as pointed out by Stam [8], though a classification and quantification of such vulnerabilities still awaits.

\section{References}

1. Bellare, M., Kohno, T.: Hash Function Balance and Its Impact on Birthday Attacks. In: Cachin, C., Camenisch, J.L. (eds.) EUROCRYPT 2004. LNCS, vol. 3027, pp. 401-418. Springer, Heidelberg (2004)

2. Black, J., Cochran, M., Shrimpton, T.: On the Impossibility of Highly-Efficient Blockcipher-Based Hash Functions. In: Cramer, R. (ed.) EUROCRYPT 2005. LNCS, vol. 3494, pp. 526-541. Springer, Heidelberg (2005)

3. Chung, F., Lu, L.: Concentration Inequalities and Martingale Inequalities: A Survey. Internet Mathematics 3(1), 79-127

4. McDiarmid, C.: Concentration. In: Habib, M., McDiarmid, C., Ramier-Alfonsin, J., Reed, B. (eds.) Probabilistic Methods for Algorithmic Discrete Mathematics. Algorithms and Combinatorics, vol. 16, pp. 195-248. Springer (1998)

5. Rogaway, P., Steinberger, J.: Constructing Cryptographic Hash Functions from Fixed-Key Blockciphers. In: Wagner, D. (ed.) CRYPTO 2008. LNCS, vol. 5157, pp. 433-450. Springer, Heidelberg (2008)

6. Rogaway, P., Steinberger, J.: Security/Efficiency Tradeoffs for Permutation-Based Hashing. In: Smart, N.P. (ed.) EUROCRYPT 2008. LNCS, vol. 4965, pp. 220-236. Springer, Heidelberg (2008)

7. Shrimpton, T., Stam, M.: Building a Collision-Resistant Compression Function from Non-compressing Primitives. In: Aceto, L., Damgård, I., Goldberg, L.A., Halldórsson, M.M., Ingólfsdóttir, A., Walukiewicz, I. (eds.) ICALP 2008, Part II. LNCS, vol. 5126, pp. 643-654. Springer, Heidelberg (2008); Also available at the Cryptology ePrint Archive: Report 2007/409

8. Stam, M.: Beyond Uniformity: Better Security/Efficiency Tradeoffs for Compression Functions. In: Wagner, D. (ed.) CRYPTO 2008. LNCS, vol. 5157, pp. 397-412. Springer, Heidelberg (2008) 
9. Steinberger, J.: Stam's Collision Resistance Conjecture. In: Gilbert, H. (ed.) EUROCRYPT 2010. LNCS, vol. 6110, pp. 597-615. Springer, Heidelberg (2010)

10. Wiener, M.: Bounds on birthday attack times. Cryptology ePrint archive (2005)

11. Wu, H.: The JH hash function. NIST SHA-3 competition submission (October 2008)

\section{A Supporting Lemmas}

We recall that for random variables $X, Y$, a notation such as $\operatorname{Var}(X \mid Y=s)$ means the variance of $X$ conditioned on the event $Y=s$, whereas $\operatorname{Var}(X \mid Y)$ is a function from the range of $Y$ to $\mathbb{R}$, that assigns $\operatorname{Var}(X \mid Y=s)$ to each $s$ in the range of $Y$ (or more precisely, to each $s$ such that $\operatorname{Pr}[Y=s]$ is nonzero). The notation "Var$(X \mid Y) \leq c$ " indicates this function is upper bounded by $c$ : $\operatorname{Var}(X \mid Y=s) \leq c$ for all $s$ such that $\operatorname{Pr}[Y=s]>0$.

We use, as a starting point, the following concentration result for Martingales. See Theorem 6.1 of [3] for a proof. (We note that our notation is slightly modified from standard in order to avoid discussion of filters.)

Lemma 2 (Folklore [3, 4]). Let $Y_{1}, \ldots, Y_{n}$ be a sequence of random variables of range $R, f: R^{n} \rightarrow \mathbb{R}$ be a function and let $Y=f\left(Y_{1}, \ldots, Y_{n}\right)$. Let

$$
X_{i}=E\left[Y \mid Y_{1}, \ldots, Y_{i}\right]
$$

for $0 \leq i \leq n$. Then if

1. $\operatorname{Var}\left(X_{i} \mid Y_{1}, \ldots, Y_{i-1}\right) \leq \sigma_{i}^{2}$ for $1 \leq i \leq n$, and

2. $\left|X_{i}-X_{i-1}\right| \leq M$, for every $1 \leq i \leq n$,

we have

$$
\operatorname{Pr}[Y \leq E[Y]-\lambda] \leq e^{-\frac{\lambda^{2}}{2\left(\sum_{i=1}^{n} \sigma_{i}^{2}+M \lambda / 3\right)}}
$$

for any $\lambda \geq 0$.

Lemma 3. Let $k, q$ be integers such that $1 \leq q \leq k$. Let $\mathcal{B}$ be random a subset of $[k]=\{1, \ldots, k\}$ of size $q$. Let $M$ and $c_{1}, \ldots, c_{k}$ be nonnegative constants such that $M \geq c_{i}$ for $1 \leq i \leq k$. Put $Y=\sum_{i \in \mathcal{B}} c_{i}$. Then

$$
\operatorname{Pr}[Y \leq E[Y]-t] \leq e^{-\frac{t^{2}}{2 M(3 E[Y]+t / 3)}}
$$

for all $t \geq 0$.

Proof. Note that if $q=k$ the lemma is obviously true, and so we can assume $q<k$.

We view the elements of $\mathcal{B}$ as being selected sequentially, with the $i$-th element of $\mathcal{B}$ coming uniformly at random from a set of size $k-i+1$ (the complement of the currently selected elements). Let $s_{i}$ be the $i$-th chosen element, and define $f:[k]^{q} \rightarrow \mathbb{R}$ by $f\left(s_{1}, \ldots, s_{q}\right)=\sum_{i=1}^{q} c_{s_{i}}$. Note $Y=f\left(s_{1}, \ldots, s_{q}\right)$. In view of applying Lemma 2 (with $Y_{i}=s_{i}$ ), we define

$$
X_{i}=E\left[Y \mid s_{1}, \ldots, s_{i}\right]
$$

for $0 \leq i \leq q$. Thus, $X_{i}$ is the expected "value" of $\mathcal{B}$ after the first $i$ elements have been chosen. 
Note that for any values $t_{1}, \ldots, t_{q} \in[k]$ and $t_{i}^{\prime} \in[k]$,

$$
\left|f\left(t_{1}, \ldots, t_{q}\right)-f\left(t_{1}, \ldots, t_{i-1}, t_{i}^{\prime}, t_{i+1}, \ldots, t_{q}\right)\right| \leq M
$$

That is, changing the $i$-th input of $f$ (i.e. the $i$-th element chosen) can only change $f$ 's output by $M$, at most. It follows (by a short but standard argument) that $\left|X_{i}-X_{i-1}\right| \leq M$ for $1 \leq i \leq q$.

We next want to upper bound $\operatorname{Var}\left(X_{i+1} \mid s_{1}, \ldots, s_{i}\right)$ independently of $s_{1}, \ldots, s_{i}$. We have:

$$
\begin{aligned}
X_{i+1} & =c_{s_{i+1}}+\frac{q-i}{k-i-1} \sum_{h \notin\left\{s_{1}, \ldots, s_{i+1}\right\}} c_{h}+\sum_{j=1}^{i} c_{s_{j}} \\
& =c_{s_{i+1}}\left(1-\frac{q-i}{k-i-1}\right)+\frac{q-i}{k-i-1} \sum_{h \notin\left\{s_{1}, \ldots, s_{i}\right\}} c_{h}+\sum_{j=1}^{i} c_{s_{j}} \\
& =c_{s_{i+1}}\left(1-\frac{q-i}{k-i-1}\right)+K
\end{aligned}
$$

where $K$ is a constant depending only on $s_{1}, \ldots, s_{i}$. Therefore,

$$
\begin{aligned}
\operatorname{Var}\left(X_{i+1} \mid s_{1}, \ldots, s_{i}\right) & =\left(1-\frac{q-i}{k-i-1}\right)^{2} \cdot \operatorname{Var}\left(c_{s_{j}} \mid s_{1}, \ldots s_{i}\right) \\
& \leq\left(1-\frac{q-i}{k-i-1}\right)^{2} \cdot \frac{1}{k-i} \sum_{j \notin\left\{s_{1}, \ldots, s_{i}\right\}} c_{j}^{2} \\
& \leq \frac{(k-q)^{2}}{(k-i)(k-i-1)^{2}} \sum_{j=1}^{k} c_{j}^{2} .
\end{aligned}
$$

We set $\sigma_{i+1}^{2}$ to this last expression, $0 \leq i<q$, so that $\operatorname{Var}\left(X_{i+1} \mid s_{1}, \ldots, s_{i}\right) \leq$ $\sigma_{i+1}^{2}$.

Let $p=q / k$. Note that $p<1$ since we are assuming $q<k$ and that $X_{0}=$ $E[Y]=p \sum_{i=1}^{k} c_{i}$. We have

$$
\begin{aligned}
\sum_{i=1}^{q} \sigma_{i}^{2} & =\sum_{j=1}^{k} c_{j}^{2} \cdot(k-q)^{2} \sum_{i=0}^{q-1} \frac{1}{(k-i)(k-i-1)^{2}} \\
& \leq \sum_{j=1}^{k} c_{j}^{2} \cdot(k-q)^{2} \sum_{i=1}^{q} \frac{1}{(k-i)^{3}} \\
& =\sum_{j=1}^{k} c_{j}^{2} \cdot(k-q)^{2}\left(\sum_{i=0}^{q-1} \frac{1}{(k-i)^{3}}+\frac{1}{(k-q)^{3}}-\frac{1}{k^{3}}\right) \\
& \leq \sum_{j=1}^{k} c_{j}^{2} \cdot(k-q)^{2}\left(\int_{0}^{q} \frac{1}{(k-x)^{3}} d x+\frac{1}{(k-q)^{3}}-\frac{1}{k^{3}}\right)
\end{aligned}
$$




$$
\begin{aligned}
& =\sum_{j=1}^{k} c_{j}^{2} \cdot\left((k-q)^{2} \cdot \frac{1}{2}\left(\frac{1}{(k-q)^{2}}-\frac{1}{k^{2}}\right)+\frac{1}{k-q}-\frac{(k-q)^{2}}{k^{3}}\right) \\
& =\left(\frac{1}{2}\left(1-\frac{(k-q)^{2}}{k^{2}}\right)+\frac{1}{k-q}-\frac{(k-q)^{2}}{k^{3}}\right) \sum_{j=1}^{k} c_{j}^{2} \\
& =\left(\frac{1}{2}\left(1-(1-p)^{2}\right)+\frac{1}{k(1-p)}-\frac{(1-p)^{2}}{k}\right) \sum_{j=1}^{k} c_{j}^{2} \\
& \leq\left(\frac{1}{2}\left(2 p-p^{2}\right)+\frac{1}{k(1-p)}\right) \sum_{j=1}^{k} c_{j}^{2} \\
& =p\left(\frac{1}{2}(2-p)+\frac{1}{q(1-p)}\right) \sum_{j=1}^{k} c_{j}^{2} \\
& =p\left(\frac{1}{2}(2-p)+\frac{1}{q}+\frac{1}{k(1-p)}\right) \sum_{j=1}^{k} c_{j}^{2} \\
& \leq p\left(\frac{1}{2}(2-p)+\frac{1}{q}+\frac{1}{k \frac{1}{k}}\right) \sum_{j=1}^{k} c_{j}^{2} \\
& \leq 3 p \sum_{j=1}^{k} c_{j}^{2} \\
& \leq 3 p \sum_{j=1}^{k} c_{j} M \\
& \leq Y \\
& \leq
\end{aligned}
$$

Then by Lemma 2, we have

$$
\operatorname{Pr}[Y<E[Y]-t] \leq e^{-\frac{t^{2}}{2\left(\sum_{i=1}^{q} \sigma_{i}^{2}+M t / 3\right)}} \leq e^{-\frac{t^{2}}{2(3 E[Y] M+M t / 3)}}=e^{-\frac{t^{2}}{2 M(3 E[Y]+t / 3)}} .
$$

Lemma 4. Let $k, q$ be integers such that $1 \leq q \leq k$. Let $\mathcal{B}$ be random a subset of $[k]=\{1, \ldots, k\}$ of size $q$. Let $M$ and $c_{1}, \ldots, c_{k}$ be nonnegative constants such that $M \geq c_{i}$ for $1 \leq i \leq k$. Put $Y=\sum_{i \in \mathcal{B}} c_{i}$. Then

$$
\operatorname{Pr}[Y<\phi-t] \leq e^{-\frac{t^{2}}{2 M(3 \phi+t / 3)}}
$$

for any $t, \phi$ such that $0 \leq t \leq \phi \leq E[Y]$.

Proof. Let $u=E[Y]-\phi$. Then by Lemma 3 .

$$
\begin{aligned}
\operatorname{Pr}[Y<\phi-t] & =\operatorname{Pr}[Y<E[Y]-u-t] \\
& \leq e^{-\frac{(t+u)^{2}}{2 M(3(u+\phi)+(t+u) / 3)}}
\end{aligned}
$$

Let $f(u)=(t+u)^{2}, g(u)=3(u+\phi)+(t+u) / 3$, we find that 


$$
\begin{aligned}
\left(\frac{f(u)}{g(u)}\right)^{\prime} \geq 0 & \Longleftrightarrow f^{\prime}(u) g(u) \geq g^{\prime}(u) f(u) \\
& \Longleftrightarrow 2 g(u) \geq g^{\prime}(u)(t+u) \\
& \Longleftrightarrow 2 g(u) \geq(3+1 / 3)(t+u) \\
& \Longleftrightarrow g(u) \geq(3+1 / 3)(t+u) \\
& \Longleftrightarrow 3(u+\phi)+(t+u) / 3 \geq(3+1 / 3)(t+u) \\
& \Longleftrightarrow 3(u+t)+(t+u) / 3 \geq(3+1 / 3)(t+u)
\end{aligned}
$$

where we use $\phi \geq t$ for the last implication. Thus (5), considered as a function of $u$ and restricted to $u \geq 0$, takes its maximum at $u=0$, which establishes (4).

Lemma 5. Let $k, q$ be integers such that $1 \leq q \leq k$ and such that $q$ is even. Let $M>0$ be a constant and let $T$ be the disjoint union of sets $T_{1}, \ldots, T_{k}$ such that $\left|T_{i}\right| \leq M$ for $1 \leq i \leq k$. Let $F: T \rightarrow U$ be some function and let

$$
C_{i}=\mid\left\{x \in T_{i}: \exists y \in T, y \neq x \text {, s.t. } F(x)=F(y)\right\} \mid
$$

Let $C=C_{1}+\cdots+C_{k}$. Let $\mathcal{B}$ be a random subset of $[k]$ of size $q$. Let

$$
\overline{C_{i}}=\left|\left\{x \in T_{i}: \exists y \in T_{j}, j \in \mathcal{B}, y \neq x, F(x)=F(y)\right\}\right|
$$

and let

$$
\bar{C}=\sum_{i: i \in \mathcal{B}} \overline{C_{i}}
$$

then

$$
\operatorname{Pr}[\bar{C}<t] \leq 2 e^{-\frac{t}{16 M}}
$$

where $t=\frac{1}{20} p^{2} C$ and $p=q / k$.

Proof. We use the following equivalent selection process for $\mathcal{B}$ : we first select, independently and uniformly at random, two subsets $L$ and $R$ of $[k]$ of size $q / 2$ each, then select an additional set $H$ of size $q-|L \cup R|$ uniformly at random from $[k]$, and finally set $\mathcal{B}=L \cup R \cup H$. Clearly, this process yields a set $\mathcal{B}$ of size $q$ that is uniformly distributed at random among all subsets of $[k]$ of size $q$.

Define random variables $Y_{1}, \ldots, Y_{k}$ by putting $Y_{i}=C_{i}$ if $i \in L, Y_{i}=0$ otherwise. Let $Y=\sum_{i=1}^{k} Y_{i}$. We have $\left|Y_{i}\right| \leq\left|T_{i}\right| \leq M$. Note that $E[Y]=$ $\frac{q / 2}{k} C=\frac{1}{2} p C$. Let $t_{0}=\frac{1}{4} p C=\frac{1}{2} E[Y]$. By Lemma 3,

$$
\begin{aligned}
\operatorname{Pr}\left[Y<E[Y]-t_{0}\right] & \leq e^{-\frac{t_{0}^{2}}{2 M\left(3 E[Y]+t_{0} / 3\right)}} \\
& =e^{-\frac{p^{2} C^{2} / 16}{2 M(3 p C / 2+p C / 12)}} \\
& =e^{-\frac{p C}{32 M(3 / 2+1 / 12)}} \\
& \leq e^{-\frac{p C}{51 M}}
\end{aligned}
$$

For the rest of the proof we assume that $Y \geq E[Y]-t_{0}=\frac{1}{4} p C$. For $1 \leq i \leq k$, let

$$
C_{i}^{L}=\mid\left\{x \in T_{i}: \exists y \in T_{j}, j \in L, y \neq x \text {, s.t. } F(x)=F(y)\right\} \mid .
$$


Recall that $t=\frac{1}{20} p^{2} C$. It is not difficult to see that if $\sum_{i \in[k]} C_{i}^{L} \leq \sum_{i \in L} C_{i}-t$, then

$$
\sum_{i \in L} \mid\left\{x \in T_{i}: \exists y \in T_{j}, j \in L, y \neq x \text {, s.t. } F(x)=F(y)\right\} \mid \geq t+1 \text {, }
$$

implying that $\bar{C} \geq t$. We can therefore assume that $\sum_{i \in[k]} C_{i}^{L} \geq \sum_{i \in L} C_{i}-t \geq$ $\frac{1}{4} p C-t$.

Define random variables $Z_{1}, \ldots, Z_{k}$ by putting $Z_{i}=C_{i}^{L}$ if $i \in R, Z_{i}=0$ otherwise, and let $Z=\sum_{i=1}^{k} Z_{i}$. Then $\left|Z_{i}\right| \leq\left|T_{i}\right| \leq M$ for all $i$. Let $\phi=$ $(p / 2)\left(\frac{1}{4} p C-t\right) \leq E[Z]$ (the latter equality follows from the fact that each set is added to $R$ with probability $p / 2$, and from the fact that $\left.\sum_{i \in[k]} C_{i}^{L} \geq \frac{1}{4} p C-t\right)$. Then

$$
\phi=\frac{1}{8} p^{2} C-\frac{1}{2} p t=\left(2.5-\frac{1}{2} p\right) t \geq 2 t .
$$

Since $0 \leq t \leq \phi \leq E[Z], t \leq \phi-t$, and $\phi \leq 2.5 t$, we have by Lemma 4 that

$$
\begin{aligned}
\operatorname{Pr}[Z<t] & \leq \operatorname{Pr}[Z<\phi-t] \\
& \leq e^{-\frac{t^{2}}{2 M(3 \phi+t / 3)}} \\
& \leq e^{-\frac{t^{2}}{2 M(3 \cdot 2.5 t+t / 3)}} \\
& =e^{-\frac{t}{M(15+2 / 3)}} \\
& \leq e^{-\frac{t}{16 M}}
\end{aligned}
$$

Since $\bar{C} \geq Z$ and since $e^{-\frac{t}{16 M}}=e^{-\frac{p^{2} C}{20 \cdot 16 M}} \geq e^{-\frac{p^{2} C}{51 M}} \geq e^{-\frac{p C}{51 M}}$, a sum bound on the two bad events (these being the event that either $Y<E[Y]-t_{0}$, or that $Z<t$ ) concludes the lemma.

Lastly, Lemma 6 below notes an following elementary result related to refinements of a set of disjoint sets, defined next.

Definition 2. Let $U_{1}, \ldots, U_{\ell}$ be a collection of finite disjoint sets. Another collection $T_{1}, \ldots, T_{k}$ of finite disjoint sets is a refinement of $U_{1}, \ldots, U_{\ell}$ if $\bigcup_{i=1}^{k} T_{i}=$ $\bigcup_{i=1}^{\ell} U_{i}$ and if either $T_{i} \subseteq U_{j}$ or $T_{i} \cap U_{j}=\emptyset$ for all $1 \leq i \leq k, 1 \leq j \leq \ell$.

Lemma 6. Let $U_{1}, \ldots, U_{\ell}$ be disjoint finite sets. Let $M \geq 1$ be a positive integer upper bounding the average size of the $U_{i}$ 's. (That is, $M \geq\left(\sum_{i}\left|U_{i}\right|\right) / \ell$.) Then there exists a refinement $T_{1}, \ldots, T_{k}$ of the sets $U_{1}, \ldots, U_{\ell}$ such that $\left|T_{i}\right| \leq M$ for all $i$ and such that $k \leq 2 \ell$.

Proof. We can refine each set $U_{i}$ into at most $\left\lceil\frac{\left|U_{i}\right|}{M}\right\rceil$ sets of size at most $M$ each 8 . Thus we can find a refinement $T_{1}, \ldots, T_{k}$ of $U_{1}, \ldots, U_{\ell}$ where $\left|T_{i}\right| \leq M$ for all $1 \leq i \leq k$ and where

$$
k \leq \sum_{i=1}^{\ell}\left\lceil\frac{\left|U_{i}\right|}{M}\right\rceil \leq \sum_{i=1}^{\ell}\left(\frac{\left|U_{i}\right|}{M}+1\right) \leq 2 \ell .
$$

\footnotetext{
${ }^{8}$ Note this actually requires $M$ to be an integer.
} 